\title{
Numerical Simulation of an Electroweak Oscillon
}

\author{
N. Grahan*
}

\author{
Department of Physics, Middlebury College, Middlebury, VT 05753
}

\begin{abstract}
Numerical simulations of the bosonic sector of the $S U(2) \times U(1)$ electroweak Standard Model in $3+1$ dimensions have demonstrated the existence of an oscillon - an extremely long-lived, localized, oscillatory solution to the equations of motion - when the Higgs mass is equal to twice the $W^{ \pm}$ boson mass. It contains total energy roughly $30 \mathrm{TeV}$ localized in a region of radius $0.05 \mathrm{fm}$. A detailed description of these numerical results is presented.

PACS numbers: 11.27.+d 11.15.Ha 12.15.-y
\end{abstract}

\section{INTRODUCTION}

While static, localized soliton solutions to the equations of motion of nonlinear field theories have been well studied, and are of interest in many applications [1, 2], no known examples exist in the electroweak Standard Model (although there do exist extended electroweak string solutions 3, 4]). However, much less is known about the existence of localized solutions that oscillate in time, known as breathers or oscillons. (The latter term was originally introduced to describe similar phenomena in plasma physics [5].) In some models, such as the sine-Gordon breather [ 6$]$ and $Q$-ball [7], one can use conserved charges to prove the existence of exact, periodic solutions. But oscillons have also been found in many nonlinear field theories that do not contain either static solitons or conserved charges. These solutions either live indefinitely or for extremely long times compared to the natural timescales of the system.

For scalar theories in one space dimension, oscillons have been found to remain periodic to all orders in a perturbative expansion [6] and are never seen to decay in numerical simulations [8], but can decay after extremely long times via nonperturbative effects [9] or by coupling to an expanding background [10]. In both $\phi^{4}$ theory in two dimensions [11, 12] and the abelian Higgs model in one dimension [13] and in two dimensions [14], oscillons have been found that are not observed to decay. In $\phi^{4}$ theory in three dimensions, however, one finds long-lived quasi-periodic solutions whose lifetime depends sensitively on the initial conditions [15, 16, 17, 18, 19]. Similar behavior is present in other scalar theories in three dimensions 20 and in higher dimensions 21]. Phenomenologically, small $Q$-balls were considered as dark matter candidates in [22, 23, 24, 25], axion oscillons were considered in 26], and the effects of oscillons and other aspects of nonequilibrium dynamics in and after inflation were studied in 27, 28, 29]. Oscillons and related solutions have also been studied in connection with phase transitions [30], monopole systems [31], QCD [32], and gravitational systems [33].

Recent work [34] demonstrated numerically the existence of an oscillon in the bosonic sector of the electroweak Standard Model, when the mass of the fundamental Higgs is exactly twice that of the $W^{ \pm}$gauge bosons. (A similar mass relation also arises in the study of embedded defects [35].) This result was based on previous work [36], which found oscillons in spontaneously broken pure $S U(2)$ Higgs-gauge theory with the same $2: 1$ mass ratio. In that model, one can consider field configurations restricted to the spherical ansatz [37], meaning they are assumed to be invariant under combined rotations in space and isospin, also known as grand spin rotations. Within this ansatz, the system can be described by an effective theory of fields depending only on $r$ and $t$, which greatly simplifies the numerical analysis. In [34] this numerical simulation was extended to a fully three-dimensional spatial lattice with no assumptions of rotational symmetry, making it possible to also include the $U(1)$ hypercharge field (which breaks the grand spin invariance of the spherical ansatz). The resulting simulation comprises the full electroweak sector of the Standard Model without fermions. Here we extend that analysis and describe its results in more detail. We use the same $S U(2)$ gauge coupling $g$ and Higgs self-coupling $\lambda$ as in the pure $S U(2)$ theory, meaning that the Higgs mass is twice the mass of the $W^{ \pm}$bosons, and set the $U(1)$ coupling $g^{\prime}$ so that the mass of the $Z^{0}$ boson matches its observed value.

Ongoing analytic work [38] has shed some light on the $2: 1$ mass ratio by using a small amplitude approximation $[6,10,32,39]$ to construct oscillons in a simplified version of the spherical ansatz theory. In this analysis, one begins by assuming that each field in the oscillon profile has large width, so that at large distances it falls like $\exp (-\epsilon m r)$, where $m$ is its mass. There, the amplitude is small and the oscillations obey a linear dispersion relation, which implies $\omega=m \sqrt{1-\epsilon^{2}}$. The linear, dispersive gradient terms in the equation of motion are then of order $\epsilon^{2}$. They must be balanced by nonlinear terms to obtain a stable solution. Since the leading nonlinearity is typically given by a quadratic term in the equations of motion, this requirement implies that the field amplitudes must be proportional 
to $\epsilon$. In a multiple-field model, one must also ensure that the terms giving interactions between different fields are resonant with the dispersive linear terms, so that their effects are not washed out over many cycles. As shown in [38], the $2: 1$ mass ratio arises naturally in this analysis: Since the fields' oscillation frequencies are tied to their masses, imposing a resonance condition on their frequencies is equivalent to fixing a particular mass ratio. Although this analysis has so far only been carried out in simplified models, we will see below that the oscillon observed numerically in the full electroweak theory is of small amplitude and large width, so similar techniques are potentially applicable in this case as well.

In all known oscillons, each field oscillates with a frequency below its mass, so that it couples to dispersive linear waves (which have $\omega=\sqrt{k^{2}+m^{2}}>m$ ) only through nonlinear interactions. The fields then converge to a configuration in which this decay channel is also suppressed. Because the electroweak theory includes the massless photon field, which can radiate in arbitrarily low frequencies, one might expect the oscillon to decay rapidly by emitting electromagnetic radiation, but it does not. Instead, after initially shedding some energy in this way, the system settles into a localized solution that no longer radiates and remains stable for as long as we can follow it in numerical simulations. In preliminary work that provided motivation for the current investigation, similar behavior was observed both when an additional massless scalar field was coupled to oscillons in one-dimensional $\phi^{4}$ theory and when an additional spherically symmetric massless scalar field was coupled to oscillons in the spherical ansatz model. In each case, after shedding some energy into the massless field, the oscillon arranges itself in a neutral configuration that no longer couples to the massless field. This mechanism may be similar to the suppression of nonlinear coupling to dispersive waves that is common to all oscillons.

\section{CONTINUUM THEORY}

We begin from $S U(2) \times U(1)$ electroweak theory in the continuum, ignoring fermions, and follow the conventions of [40]. The Lagrangian density is

$$
\mathcal{L}=-\frac{1}{4} F_{\mu \nu} F^{\mu \nu}-\frac{1}{4} \boldsymbol{F}_{\mu \nu} \cdot \boldsymbol{F}^{\mu \nu}+\left(D_{\mu} \Phi\right)^{\dagger} D^{\mu} \Phi-\lambda\left(|\Phi|^{2}-v^{2}\right)^{2},
$$

where the boldface vector notation refers to isovectors. Here $\Phi$ is the Higgs field, a Lorentz scalar carrying $U(1)$ hypercharge $1 / 2$ and transforming under the fundamental representation of $S U(2)$. The metric signature is +--- . The $S U(2)$ and $U(1)$ field strengths are

$$
\boldsymbol{F}_{\mu \nu}=\partial_{\mu} \boldsymbol{W}_{\nu}-\partial_{\nu} \boldsymbol{W}_{\mu}-g \boldsymbol{W}_{\mu} \times \boldsymbol{W}_{\nu}, \quad F_{\mu \nu}=\partial_{\mu} B_{\nu}-\partial_{\nu} B_{\mu},
$$

and the covariant derivatives are given by

$$
D_{\mu} \Phi=\left(\partial_{\mu}+i \frac{g^{\prime}}{2} B_{\mu}+i \frac{g}{2} \boldsymbol{\tau} \cdot \boldsymbol{W}_{\mu}\right) \Phi, \quad D^{\mu} \boldsymbol{F}_{\mu \nu}=\partial^{\mu} \boldsymbol{F}_{\mu \nu}-g \boldsymbol{W}^{\mu} \times \boldsymbol{F}_{\mu \nu}
$$

where $\tau$ represents the weak isospin Pauli matrices. We obtain the equations of motion

$$
\partial_{\mu} F^{\mu \nu}=J^{\nu}, \quad D_{\mu} \boldsymbol{F}^{\mu \nu}=\boldsymbol{J}^{\nu}, \quad D^{\mu} D_{\mu} \Phi=2 \lambda\left(v^{2}-|\Phi|^{2}\right) \Phi
$$

where the gauge currents are

$$
J_{\nu}=g^{\prime} \operatorname{Im}\left(D_{\nu} \Phi\right)^{\dagger} \Phi, \quad \boldsymbol{J}_{\nu}=g \operatorname{Im}\left(D_{\nu} \Phi\right)^{\dagger} \boldsymbol{\tau} \Phi
$$

We work in the gauge $B_{0}=0, \boldsymbol{W}_{0}=\mathbf{0}$. With this choice, the covariant time derivatives become ordinary derivatives and we can apply a Hamiltonian formalism. The energy density is

$$
u=\frac{1}{2} \sum_{j=x, y, z}\left[\dot{B}_{j}^{2}+\dot{\boldsymbol{W}}_{j} \cdot \dot{\boldsymbol{W}}_{j}+\sum_{k>j}\left(F_{k j}^{2}+\boldsymbol{F}_{k j} \cdot \boldsymbol{F}_{k j}\right)\right]+|\dot{\Phi}|^{2}+\sum_{j=x, y, z}\left(D_{j} \Phi\right)^{\dagger}\left(D_{j} \Phi\right)+\lambda\left(|\Phi|^{2}-v^{2}\right)^{2}
$$

where dot indicates time derivative. The integral over space of this quantity is conserved by the time evolution. From the equations for $B_{0}$ and $\boldsymbol{W}_{0}$, we obtain the Gauss's Law constraints,

$$
\sum_{j=x, y, z} \partial_{j} \dot{B}_{j}-J_{0}=0, \quad \sum_{j=x, y, z} D_{j} \dot{\boldsymbol{W}}_{j}-\boldsymbol{J}_{0}=0
$$


where the charge densities are

$$
J_{0}=g^{\prime} \operatorname{Im} \dot{\Phi}^{\dagger} \Phi, \quad \boldsymbol{J}_{0}=g \operatorname{Im} \dot{\Phi}^{\dagger} \boldsymbol{\tau} \Phi
$$

These constraints remain true at all times, at all points in space, assuming they are obeyed by the initial value data.

Although the numerical calculation will be done using the underlying gauge fields $\boldsymbol{W}_{\mu}$ and $B_{\mu}$, because of spontaneous symmetry breaking the physical content of the theory is better described by the fields of definite mass and electric charge

$$
\begin{aligned}
W_{\mu}^{ \pm} & =\frac{1}{\sqrt{2}}\left[\left(\boldsymbol{W}_{\mu} \cdot \hat{\boldsymbol{x}}\right) \pm i\left(\boldsymbol{W}_{\mu} \cdot \hat{\boldsymbol{y}}\right)\right] \\
Z_{\mu}^{0} & =\left(\boldsymbol{W}_{\mu} \cdot \hat{\boldsymbol{z}}\right) \cos \theta_{W}-B_{\mu} \sin \theta_{W} \\
A_{\mu} & =B_{\mu} \cos \theta_{W}+\left(\boldsymbol{W}_{\mu} \cdot \hat{\boldsymbol{z}}\right) \sin \theta_{W}
\end{aligned}
$$

where $\hat{\boldsymbol{x}}, \hat{\boldsymbol{y}}$, and $\hat{\boldsymbol{z}}$ denote unit vectors in isospin space and $\theta_{W}=\arctan \left(g^{\prime} / g\right)$ is the weak mixing angle. The $W_{\mu}^{ \pm}$ fields have mass $m_{W}=g v / \sqrt{2}$ and electric charge $\pm e= \pm g^{\prime} \cos \theta_{W}$, the $Z_{\mu}^{0}$ field has mass $m_{Z}=m_{W} / \cos \theta_{W}$ and zero electric charge, and the photon field $A_{\mu}$ has zero mass and zero electric charge. The only other physical degree of freedom in the theory is the magnitude of the Higgs field, with mass $m_{H}=2 v \sqrt{\lambda}$ and zero electric charge.

\section{LATTICE THEORY}

To analyze the classical equations of motion numerically, we use the standard Wilsonian approach [41] for lattice gauge fields (for a review see [42]), adapted to Minkowski space evolution as in [43, 44, 45]. The $U(1)$ and $S U(2)$ gauge fields live on the links of the lattice and the Higgs field lives at the lattice sites. We use a regular lattice with spacing $\Delta x$ and determine the values of the fields at time $t_{+}=t+\Delta t$ based on their values at times $t$ and $t_{-}=t-\Delta t$. Throughout, we will use the same notation and conventions as [34].

We associate the Wilson line

$$
U_{j}^{p}=e^{i g^{\prime} B_{j}^{p} \Delta x / 2} e^{i g \boldsymbol{W}_{j}^{p} \cdot \boldsymbol{\tau} \Delta x / 2}
$$

with the link emanating from lattice site $p$ in the positive $j^{\text {th }}$ direction. We define the Wilson line for the link emanating from lattice site $p$ in the negative $j^{\text {th }}$ direction to be the adjoint of the corresponding Wilson line emanating in the positive direction from the neighboring site, $U_{-j}^{p}=\left(U_{j}^{p-j}\right)^{\dagger}$, where the notation $p \pm j$ indicates the adjacent lattice site to $p$, displaced from $p$ in direction $\pm j$. At the edges of the lattice we use periodic boundary conditions.

The equation of motion for the Higgs field at site $p$ is

$$
\Phi^{p}\left(t_{+}\right)=2 \Phi^{p}(t)-\Phi^{p}\left(t_{-}\right)+\Delta t^{2} \ddot{\Phi}^{p}(t)
$$

where

$$
\ddot{\Phi}^{p}(t)=\sum_{j= \pm x, \pm y, \pm z} \frac{U_{j}^{p}(t) \Phi^{p+j}(t)-\Phi^{p}(t)}{\Delta x^{2}}+2 \lambda\left(v^{2}-\left|\Phi^{p}(t)\right|^{2}\right) \Phi^{p}(t) .
$$

For the gauge fields, we have

$$
U_{j}^{p}\left(t_{+}\right)=\exp \left[\log U_{j}^{p}(t) U_{j}^{p}\left(t_{-}\right)^{\dagger}-\left(\sum_{j^{\prime} \neq j} \frac{\log U_{\square\left(j, j^{\prime}\right)}^{p}(t)+\log U_{\square\left(j,-j^{\prime}\right)}^{p}(t)}{\Delta x^{2}}+\frac{i \Delta x}{2}\left(g^{\prime} J_{j}^{p}+g \boldsymbol{J}_{j}^{p} \cdot \boldsymbol{\tau}\right)\right) \Delta t^{2}\right] U_{j}^{p}(t),
$$

where $U_{\square\left(j, j^{\prime}\right)}^{p}(t)=U_{j}^{p}(t) U_{j^{\prime}}^{p+j}(t) U_{-j}^{p+j+j^{\prime}}(t) U_{-j^{\prime}}^{p+j^{\prime}}(t)$ and

$$
J_{j}^{p}=g^{\prime} \operatorname{Im} \frac{\Phi^{p}(t)^{\dagger} U_{j}^{p}(t) \Phi^{p+j}(t)}{\Delta x}, \quad \boldsymbol{J}_{j}^{p}=g \operatorname{Im} \frac{\Phi^{p}(t)^{\dagger} \boldsymbol{\tau} U_{j}^{p}(t) \Phi^{p+j}(t)}{\Delta x},
$$

are the gauge currents. Here we have defined the logarithm of a $2 \times 2$ matrix in the form of Eq. (10) as

$$
\log U_{j}^{p}=\frac{i \Delta x}{2}\left(g^{\prime} B_{j}^{p}+g \boldsymbol{W}_{j}^{p} \cdot \boldsymbol{\tau}\right)
$$


which gives the more familiar gauge fields in terms of the link variables. We note that $\log X Y \neq \log X+\log Y$ when the matrices do not commute.

The $U(1)$ and $S U(2)$ matrices in Eq. (10) are stored separately in the numerical code. To represent the $U(1)$ matrix $U_{1}=e^{i \theta}$, just the real quantity $\theta=g^{\prime} B_{j}^{p} \Delta x / 2$ is actually stored. Any $S U(2)$ matrix can be written as

$$
U_{2}=\left(\begin{array}{cc}
x_{1} & x_{2} \\
-x_{2}^{*} & x_{1}^{*}
\end{array}\right)
$$

so only the two complex elements of the top row need to be stored. (This representation is redundant, since $\left|x_{1}\right|^{2}+$ $\left|x_{2}\right|^{2}=1$, but more efficient computationally than storing three real quantities and reconstructing the fourth.) The logarithms and exponentials needed to convert between the group and the algebra can be computed efficiently using

$$
U_{2}=e^{i \theta \hat{\boldsymbol{n}} \cdot \overrightarrow{\boldsymbol{\tau}}}=\cos \theta+i \hat{\boldsymbol{n}} \cdot \overrightarrow{\boldsymbol{\tau}} \sin \theta=\left(\begin{array}{cc}
\cos \theta+i \hat{\boldsymbol{n}}_{z} \sin \theta & i \hat{\boldsymbol{n}}_{x} \sin \theta+\hat{\boldsymbol{n}}_{y} \sin \theta \\
i \hat{\boldsymbol{n}}_{x} \sin \theta-\hat{\boldsymbol{n}}_{y} \sin \theta & \cos \theta-i \hat{\boldsymbol{n}}_{z} \sin \theta
\end{array}\right),
$$

where $\hat{\boldsymbol{n}}$ is a unit vector and the link matrices have $\hat{\boldsymbol{n}} \theta=\boldsymbol{W}_{j}^{p} g \Delta x / 2$.

We note that this discretization differs slightly from the standard approach used in [43, 44, 45]. In our language, their discretization is equivalent to replacing $\sin \theta \rightarrow \theta$ and $\cos \theta \rightarrow \sqrt{1-\theta^{2}}$ when computing both the logarithm and the corresponding exponential. While the approach we are using corresponds a little more directly to the continuum equations, any differences are of higher order in the lattice spacing. Numerical experiments show that their approach yields completely equivalent results, and is somewhat more efficient computationally, since it avoids the need to compute trigonometric functions in this conversion.

The energy density at $p$ is then

$$
\begin{aligned}
u^{p}(t)= & \frac{1}{2} \sum_{j=x, y, z}\left[\frac{\left\|\exp \left(\log U_{j}^{p}\left(t_{+}\right)-\log U_{j}^{p}\left(t_{-}\right)\right)\right\|^{2}}{(2 \Delta t)^{2}}+\sum_{j^{\prime}>j} \frac{\left\|U_{\square\left(j, j^{\prime}\right)}^{p}(t)\right\|^{2}}{\Delta x^{2}}\right] \\
& +\frac{\left|\Phi^{p}\left(t_{+}\right)-\Phi^{p}\left(t_{-}\right)\right|^{2}}{(2 \Delta t)^{2}}+\sum_{j=x, y, z} \frac{\left|U_{j}^{p}(t) \Phi^{p+j}(t)-\Phi^{p}(t)\right|^{2}}{\Delta x^{2}}+\lambda\left(\left|\Phi^{p}\right|^{2}-v^{2}\right)^{2},
\end{aligned}
$$

whose integral over the whole lattice is conserved. Here we have defined

$$
\left\|U_{j}^{p}\right\|^{2}=\frac{\left|\operatorname{Tr} \log U_{j}^{p}\right|^{2}}{g^{\prime 2} \Delta x^{2}}+\frac{\left(\operatorname{Tr} \boldsymbol{\tau} \log U_{j}^{p}\right)^{\dagger} \cdot\left(\operatorname{Tr} \boldsymbol{\tau} \log U_{j}^{p}\right)}{g^{2} \Delta x^{2}}=\left|B_{j}^{p}\right|^{2}+\boldsymbol{W}_{j}^{p} \cdot \boldsymbol{W}_{j}^{p}
$$

for any $U(2)$ link matrix.

At every lattice point, Gauss's Law,

$$
\sum_{j=x, y, z} \frac{\log U_{j}^{p}\left(t_{+}\right) U_{j}^{p}(t)^{\dagger}+\log U_{-j}^{p}\left(t_{+}\right) U_{-j}^{p}(t)^{\dagger}}{2 i \Delta x^{2} \Delta t}-\left(g^{\prime} J_{0}^{p}+g \boldsymbol{J}_{0}^{p} \cdot \boldsymbol{\tau}\right)=0,
$$

is also maintained throughout the evolution, where the charge densities are given by

$$
J_{0}=g^{\prime} \operatorname{Im}\left(\frac{\Phi^{p}\left(t_{+}\right)-\Phi^{p}(t)}{\Delta t}\right)^{\dagger} \Phi^{p}(t), \quad \boldsymbol{J}_{0}=g \operatorname{Im}\left(\frac{\Phi^{p}\left(t_{+}\right)-\Phi^{p}(t)}{\Delta t}\right)^{\dagger} \boldsymbol{\tau} \Phi^{p}(t) .
$$

This requirement will provide a stringent check on the correctness of the numerical simulation. Here we have computed Gauss's Law at time $t+\Delta t / 2$, which is obeyed exactly by the discrete equations of motion for any time step and lattice spacing. In [34], Gauss's Law at time $t$ was used; it is only obeyed to order $\Delta t^{2}$, but as a result it also provides a rough estimate of whether the time step is small enough.

\section{SPHERICAL ANSATZ}

With the $U(1)$ field included, the grand spin symmetry of the spherical ansatz used in [36] is broken and field configurations will not maintain this symmetry under time evolution. The continuum theory does still preserve 
invariance under grand spin rotations around the $z$-axis, but the Cartesian lattice provides a small breaking of all rotational symmetries. As a result, field configurations that start within the spherical ansatz are not constrained to lie in any reduced ansatz at later times. (We will also demonstrate the oscillon's stability under explicitly nonspherical deformations below.) Nonetheless, because we will use the spherical ansatz as a starting point to obtain our initial conditions, it will be helpful to analyze it in more detail. We will see that the electroweak oscillon retains much of the structure it inherits from these initial conditions.

For our choice of gauge, the spherical ansatz takes the form [37]

$$
\begin{aligned}
\boldsymbol{\tau} \cdot \boldsymbol{W}_{j} & =\frac{1}{g}\left[a_{1}(r, t) \boldsymbol{\tau} \cdot \hat{\boldsymbol{r}} \hat{r}_{j}+\frac{\alpha(r, t)}{r}\left(\tau_{j}-\boldsymbol{\tau} \cdot \hat{\boldsymbol{r}} \hat{r}_{j}\right)-\frac{\gamma(r, t)}{r}(\hat{\boldsymbol{r}} \times \boldsymbol{\tau})_{j}\right] \\
\Phi & =\frac{1}{g}[\mu(r, t)-i \nu(r, t) \boldsymbol{\tau} \cdot \hat{\boldsymbol{r}}]\left(\begin{array}{l}
0 \\
1
\end{array}\right),
\end{aligned}
$$

where $\boldsymbol{r}$ is the position vector, $r=|\boldsymbol{r}|$ is the distance from the origin, and $\hat{\boldsymbol{r}}=\boldsymbol{r} / r$ is the unit radial vector. Configurations in this ansatz are then described by reduced fields $a_{1}, \alpha, \gamma$, $\mu$, and $\nu$, all of which depend only on $r$ and $t$. The field definitions have been chosen so that the reduced fields match those used in [36], even though the conventions for the three-dimensional theory used here are slightly different.

These configurations are in the grand spin zero channel, meaning they are symmetric under simultaneous rotations in space and isospin. The gauge field $\boldsymbol{W}_{j}$ has isospin $i=1$ and internal angular momentum $s=1$. These two spins can be coupled together to yield total generalized angular momentum 0,1 , and 2 . To obtain grand spin $G=0$, these combinations must then be coupled with equal orbital angular momenta $\ell=0, \ell=1$, and $\ell=2$ respectively, corresponding to monopole, dipole and quadrupole spatial distributions. These three possibilities are reflected in Eq. (22) through the three terms $\alpha(r, t), \gamma(r, t)$, and $a_{1}(r, t)$.

We have written the Higgs field as a matrix times a fixed isospinor. This matrix transforms under both the gauged $S U(2)_{L}$ and global $S U(2)_{R}$ isospin transformations. (We are only considering global rotations in both cases, however.) Under both transformations it has isospin $i=1 / 2$, giving total isospin $i=0$ or $i=1$. Since the Higgs is a Lorentz scalar, with zero internal angular momentum, to obtain $G=0$ these two possibilities must be coupled to $\ell=0$ and $\ell=1$ respectively, corresponding to monopole and dipole spatial distributions. These possibilities appear in Eq. (22) as the terms $\mu(r, t)$ and $\nu(r, t)$.

Although the spherical ansatz does not contain the $U(1)$ field, to leading order in $\theta_{W}$ we can find the electric charge density created by a spherical ansatz configuration for our choice of gauge [46],

$$
J_{0}=\frac{2 e z}{r^{3} g^{2}}(\gamma \dot{\alpha}-\alpha \dot{\gamma}) .
$$

The charge shows a dipole structure centered on the $z$ axis - as we would expected since the electromagnetic interactions break the grand spin symmetry by selecting the $z$ direction in isospin. We note that this electric charge density is time independent (and thus does not radiate) if the $\alpha$ and $\gamma$ fields vary sinusoidally in time with the same frequency.

\section{NUMERICAL SIMULATION}

The initial conditions for the simulation are obtained starting from an approximate functional fit to the solutions that were found in $S U(2)$-Higgs theory using the spherical ansatz [36]. These results, with slight modifications, provide the initial data for the $\boldsymbol{W}_{j}$ and $\Phi$ fields, and the initial $B_{j}$ field is chosen to vanish. In order to guarantee that the initial configuration obeys Gauss's Law in the full $S U(2) \times U(1)$ theory, we generate the spherical ansatz fit at a point in the cycle where the time derivatives are smallest, and then set all time derivatives to zero. We note that in pure $S U(2)$ Higgs-gauge theory, this restriction would not be necessary, because even though an approximate fit with nonvanishing time derivatives will not obey Gauss's Law, we can restore Gauss's Law by adjusting $\Phi\left(t_{+}\right)$slightly via an $S U(2)$ transformation at each point,

$$
\Phi_{\text {new }}\left(t_{+}\right)=\left|\frac{\Phi_{\text {old }}\left(t_{+}\right)}{\Phi(t)}\right| \mathcal{U}^{p} \Phi(t)
$$

with

$$
\mathcal{U}^{p}=\exp \left[\sum_{j=x, y, z} \frac{\log U_{j}^{p}\left(t_{+}\right) U_{j}^{p}(t)^{\dagger}+\log U_{-j}^{p}\left(t_{+}\right) U_{-j}^{p}(t)^{\dagger}}{g^{2} \Delta x^{2}\left|\Phi_{\text {old }}\left(t_{+}\right)\right||\Phi(t)| / 2}\right]^{\dagger}
$$


This procedure has been used successfully to reproduce spherical ansatz solutions with nonvanishing time derivatives at $t=0$ in a fully three-dimensional simulation of pure $S U(2)$ Higgs-gauge theory, but it cannot be extended to the $S U(2) \times U(1)$ theory because $\Phi$ carries both charges, and thus cannot be adjusted to satisfy both constraints at once. Therefore we will consider only initial conditions in which all fields have zero time derivatives, so that Gauss's Law is trivially satisfied.

To construct the initial conditions, we begin from the spherical ansatz form of Eq. (22). We work in units where $v=1 / \sqrt{2}$. Since we are dealing with purely classical dynamics, we can rescale the fields to fix the $S U(2)$ coupling constant at $g=\sqrt{2}$, so that the $W^{ \pm}$mass is then $m_{W}=g v / \sqrt{2}=1 / \sqrt{2}$. With this rescaling, we must also introduce an overall factor of $g^{2} / g_{W}^{2}$ multiplying the total energy, where $g_{W}=0.634$ is the true weak coupling constant. (This factor was incorrectly omitted in the original version of [34].) We choose $\lambda=1$, so that the Higgs mass is twice the $W^{ \pm}$mass, $m_{H}=2 v \sqrt{\lambda}=\sqrt{2}$. Finally, we fix $g^{\prime}=0.773$, so that the ratio $g^{\prime} / g$ matches its observed value and the $Z^{0}$ boson has the correct mass. With these choices, one unit of energy is $114 \mathrm{GeV}$, one unit of time is $5.79 \times 10^{-27}$ sec, and one unit of length is $1.74 \times 10^{-18} \mathrm{~m}$. In these units, we take the following initial configuration for the radial fields,

$$
\begin{aligned}
a_{1}(r) & =\chi(0.117 \chi+0.016 \chi r)(\operatorname{sech} 2 \chi r)^{1 / 8}, \\
\mu(r) & =1-0.138 \chi \operatorname{sech} \frac{\chi r}{6.75}, \\
\nu(r) & =0.017 \chi r \operatorname{sech} \frac{\chi r}{5} \\
\alpha(r) & =0.117 \chi^{2} r \operatorname{sech} \frac{\chi r}{8}, \\
\gamma(r) & =0,
\end{aligned}
$$

where the adjustable parameter $\chi$ allows us to include a combined rescaling of the fields' amplitudes and $r$-dependence, as is commonly used in a small amplitude analysis [6, 32, 39]. While $\chi=1$ gives an approximation to the spherical ansatz solution of [36], a slightly larger value appears to be necessary for the configuration to settle into a stable solution in the full $S U(2) \times U(1)$ model. Here we will use $\chi=1.15$. The first term in parentheses in the definition of $a_{1}(r)$ is scaled with an additional $\chi$ so that it matches the coefficient of $\alpha$, ensuring that $\alpha, a_{1}-\alpha / r, \gamma / r$, and $\nu$ all vanish as $r \rightarrow 0$, as required for regularity of the fields at the origin. Within the spherical ansatz simulation, these initial conditions converge to a long-lived oscillon in the pure $S U(2)$-Higgs theory, which is never observed to decay. As a check of the numerical calculation, the full three-dimensional simulation agrees with the spherical ansatz simulation when the $U(1)$ interaction is turned off.

Although initial conditions of this form do settle into stable oscillon configurations in the $S U(2) \times U(1)$ theory, it is helpful to make a minor modification to them that is outside the spherical ansatz: setting the $\tau_{z}$-component of $\boldsymbol{W}_{j}$ to zero brings the initial conditions significantly closer to the localized solution that the fields ultimately converge to. While we obtain an equivalent oscillon solution in both cases, this modification reduces the energy shed as the oscillon forms. Doing so provides a significant technical benefit, because the radiation emitted as the configuration settles into the oscillon solution can wrap around the periodic boundary conditions, return to the region of the oscillon, and potentially destabilize it. To avoid this problem, the energy density in this radiation, which spreads throughout the volume of the simulation, must be small compared to the oscillon's energy density. As long as the lattice volume is large enough compared to the oscillon size, this radiation is sufficiently diffuse that it does not affect the oscillon's evolution. We use a lattice of size $L=144$ on a side in natural units, which is more than enough to satisfy this criterion. For $L \gtrsim 100$, changing the lattice size simply changes the pattern of noise caused by electromagnetic radiation superimposed on the oscillon region, but does not affect oscillon properties or stability. We can therefore be certain that there is no coherent structure to this unphysical radiation that could possibly be necessary for the oscillon's stability. Its only potential effect is to destabilize the oscillon, and it only does so when artificially concentrated by a small lattice (e.g. of size $L<100$ ). In numerical experiments, these destabilization effects are actually much weaker in the electroweak model than in pure scalar or $S U(2)$ Higgs-gauge models, because in the electroweak model the radiated energy ends up almost entirely in the electromagnetic field, while the oscillon arranges itself to be electrically neutral. For this reason, it is not necessary to use absorptive techniques such as adiabatic damping [11] or an expanding background [10], although both have been applied successfully to this problem as well. However, clearly it is helpful to adjust the initial conditions to be as close as possible to the true oscillon configuration, to minimize the amount of unwanted energy emitted as the configuration settles into the oscillon solution, and therefore limit the numerical costs associated with a larger lattice.

Starting from the modified spherical ansatz initial conditions, we let the system evolve for as long as is practical numerically, and see no sign of oscillon decay. We use lattice spacing $\Delta x=0.75$, though $\Delta x=0.625$ and $\Delta x=0.25$ were verified to give completely equivalent results in correspondingly smaller tests. The time step is $\Delta t=0.1$. Time steps of 0.05 and 0.025 also gave equivalent results, although in this case one must take into account the fact that 


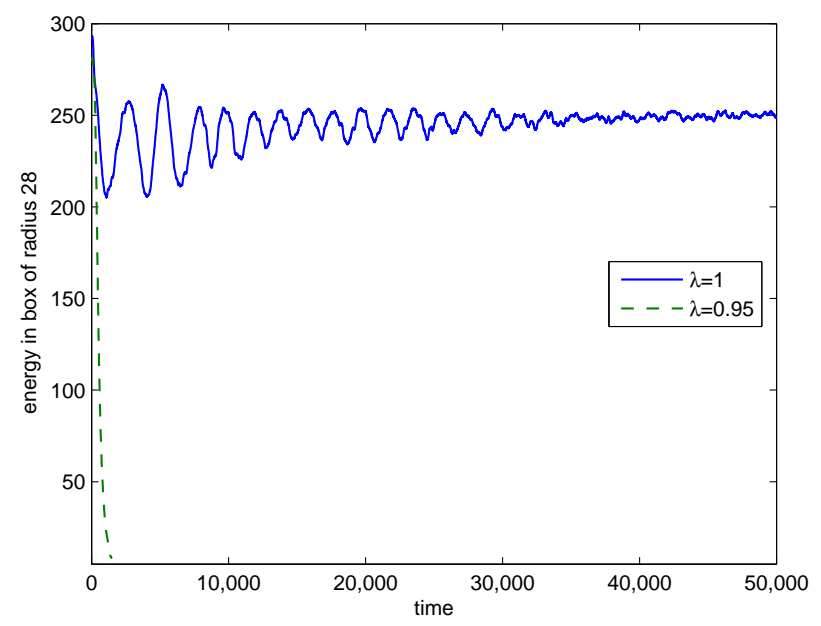

FIG. 1: Energy in a spherical box of radius 28 as a function of time in natural units. The initial conditions are given by the modified spherical ansatz form given in the text, in which the $\tau_{z}$ component of the gauge field is set to zero, with $\chi=1.15$. Two values of the Higgs self-coupling $\lambda$ are shown. For $\lambda=1$, the masses of the Higgs and $W$ fields are in the $2: 1$ ratio needed for oscillon formation and the solution remains localized throughout the simulation. Here one unit of energy is $114 \mathrm{GeV}$, one unit of time is $5.79 \times 10^{-27} \mathrm{sec}$, and one unit of length is $1.74 \times 10^{-18} \mathrm{~m}$, giving a total energy of roughly 30 TeV within the box radius of roughly $0.05 \mathrm{fm}$. A transient beat pattern is also visible. For $\lambda=0.95$, the mass ratio is $1.95: 1$. In that case, there is no stable object and the energy quickly disperses.
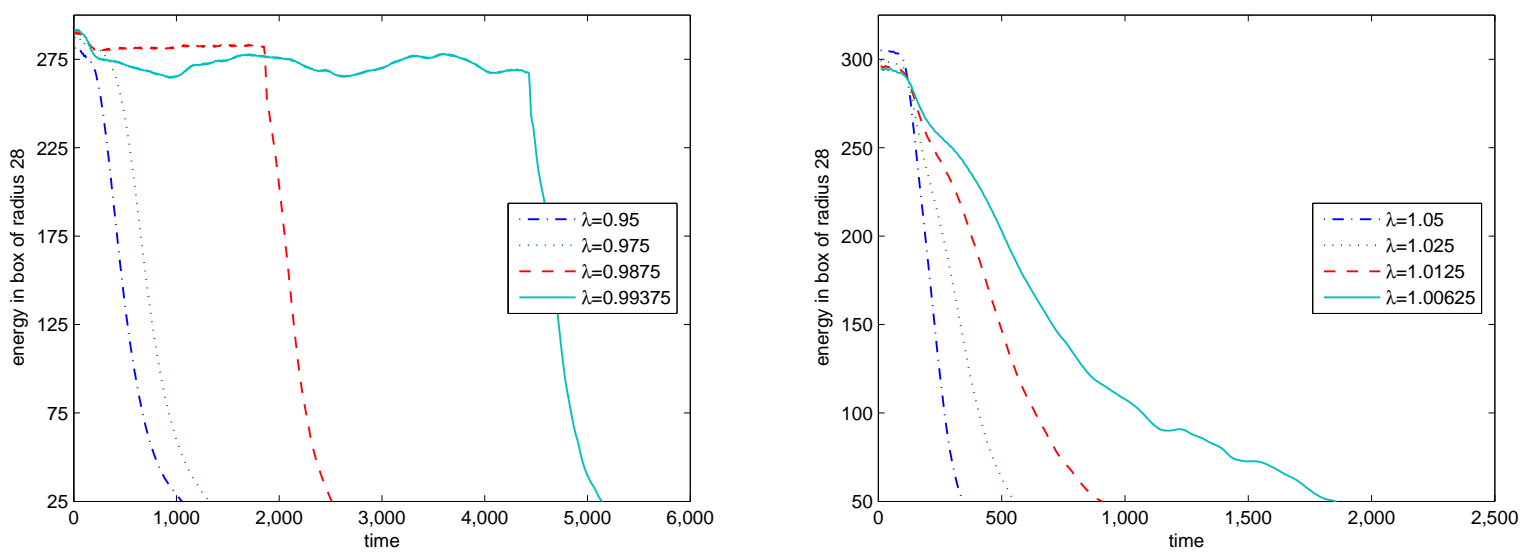

FIG. 2: Energy in the spherical box for a variety of values of $\lambda$. For $\lambda=1$, the Higgs mass is twice the $W^{ \pm}$mass and no decay is observed. When the Higgs mass is just below this value, we see a region of meta-stability. For $\lambda<1$, the fields decay by first collapsing inward before dispersing, while for $\lambda>1$ the fields simply disperse outward.

this change also slightly alters the initial conditions: To set the initial time derivatives to zero, the simulation sets the first two time slices equal. Changing the time step thus changes the time at which the field configuration matches its value at $t=0$, representing a slight perturbation of the initial conditions. This change slightly alters the initial transient behavior as the fields approach the oscillon, but these differences quickly disappear and the simulations approach equivalent oscillon configurations.

Total energy is conserved to a few parts in $10^{3}$ for $\Delta t=0.1$, which improves with $\Delta t^{2}$ as expected for our secondorder algorithm. We check Gauss's Law by monitoring the left-hand side of Eq. (20), which we verify vanishes to machine precision throughout the simulation. ${ }^{1}$ It is necessary, however, to use double precision to avoid gradual

\footnotetext{
${ }^{1}$ One can instead evaluate Gauss's Law at time $t$ instead of $t+\Delta t / 2$ as in [34]. In that case, we square the left-hand side of Eq. 20],
} 

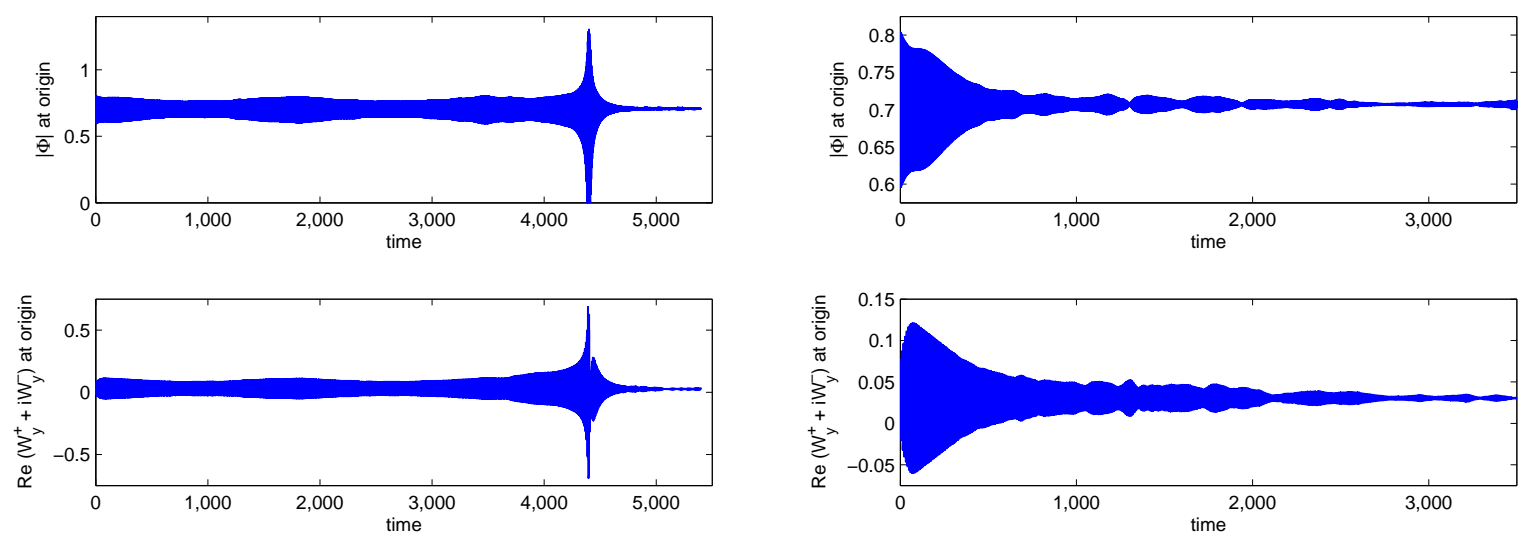

FIG. 3: Decay of the oscillon for $\lambda \neq 1$. One of the gauge fields and the magnitude of the Higgs field at the origin are shown as functions of time. In the left panel $\lambda=0.99375$ and the oscillon decays by collapsing inwards, creating a large amplitude fluctuation at the origin before dispersing. In the right, panel, $\lambda=1.00625$, and the oscillon decays by expanding outwards.

degradation in this result. For the parameters as given above, a run to time 10,000 takes roughly 40 hours using 24 parallel processes, each running on a $2 \mathrm{GHz}$ Opteron processor core. ${ }^{2}$

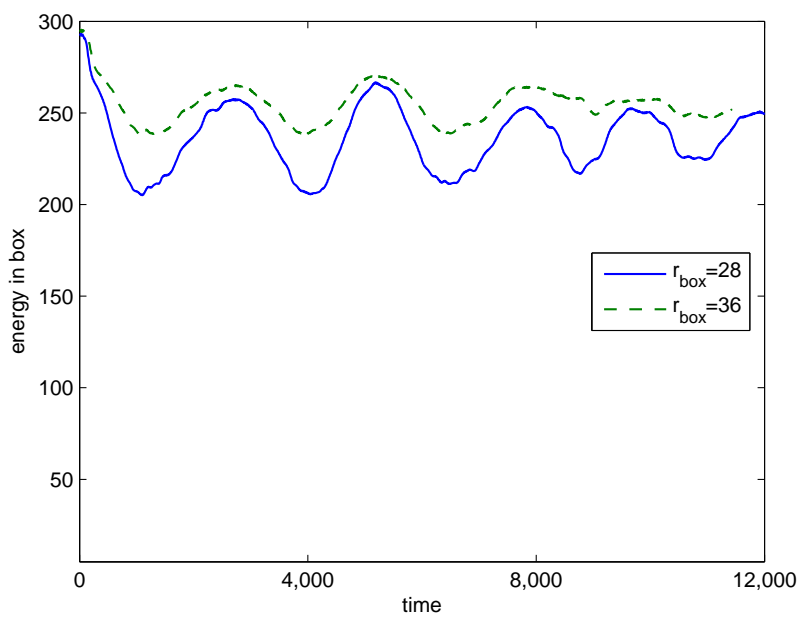

FIG. 4: Energy in the spherical box for two different box radii in the simulation of Fig. 1 with $\lambda=1$. The transient beat pattern represents a "breathing" perturbation in which the oscillon stretches and compresses slightly. For the larger box size, less energy flows in and out of the box during this process, and so the observed beat amplitude is smaller.

Fig. 1 1 shows the energy in a spherical box of radius 28 as the fields are evolved from these initial conditions. When the Higgs mass is twice the $W^{ \pm}$mass, a small amount of energy is initially emitted from the central region, with the rest remaining localized for the length of the simulation. If the masses are not in this ratio, however, the initial configuration quickly disperses. Fig. 2 shows the growth in oscillon lifetime as $\lambda$ approaches this critical value. We see a region of meta-stability when the Higgs mass is just below the $2: 1$ ratio. For $\lambda<1$, the fields first collapse toward the origin before dispersing, while for $\lambda>1$ they simply spread outward. This behavior is shown in Fig. 3 , Other "special" ratios, such as $m_{H}=2 m_{Z}$, did not form stable objects from these initial conditions.

take its trace, and then take the square root of the result. For a typical run with $\Delta t=0.1$, the integral of this quantity over the lattice never exceeds 0.025 and shows no upward trend over time. For smaller $\Delta t$, we see the expected $\mathcal{O}\left(\Delta t^{2}\right)$ improvement in this result.

2 The parallel $\mathrm{C}++$ code used for these simulations is available from http://community.middlebury.edu/ ngraham 
The spherical box contains approximately $3 \%$ of the total volume available to the simulation. Its radius has been chosen to be just large enough to enclose nearly all of energy density associated with the stable oscillon. As a result of this choice, the $\lambda=1$ graph also shows a transient beat pattern. It represents a "breathing" or "ringing" motion, in which the oscillon gradually expands and contracts slightly over many periods, accompanied by a corresponding modulation of the field amplitudes. This process causes a small amount of the oscillon's energy to move in and out of the box. As we would expect, when a larger box size is used, the "breathing" is more completely contained within the box and the graph of the energy in the box flattens out, as shown in Fig. 目 Similar beats appear in the $S U(2)$ spherical ansatz oscillon [36], but in the electroweak oscillon their amplitude decays much more rapidly.
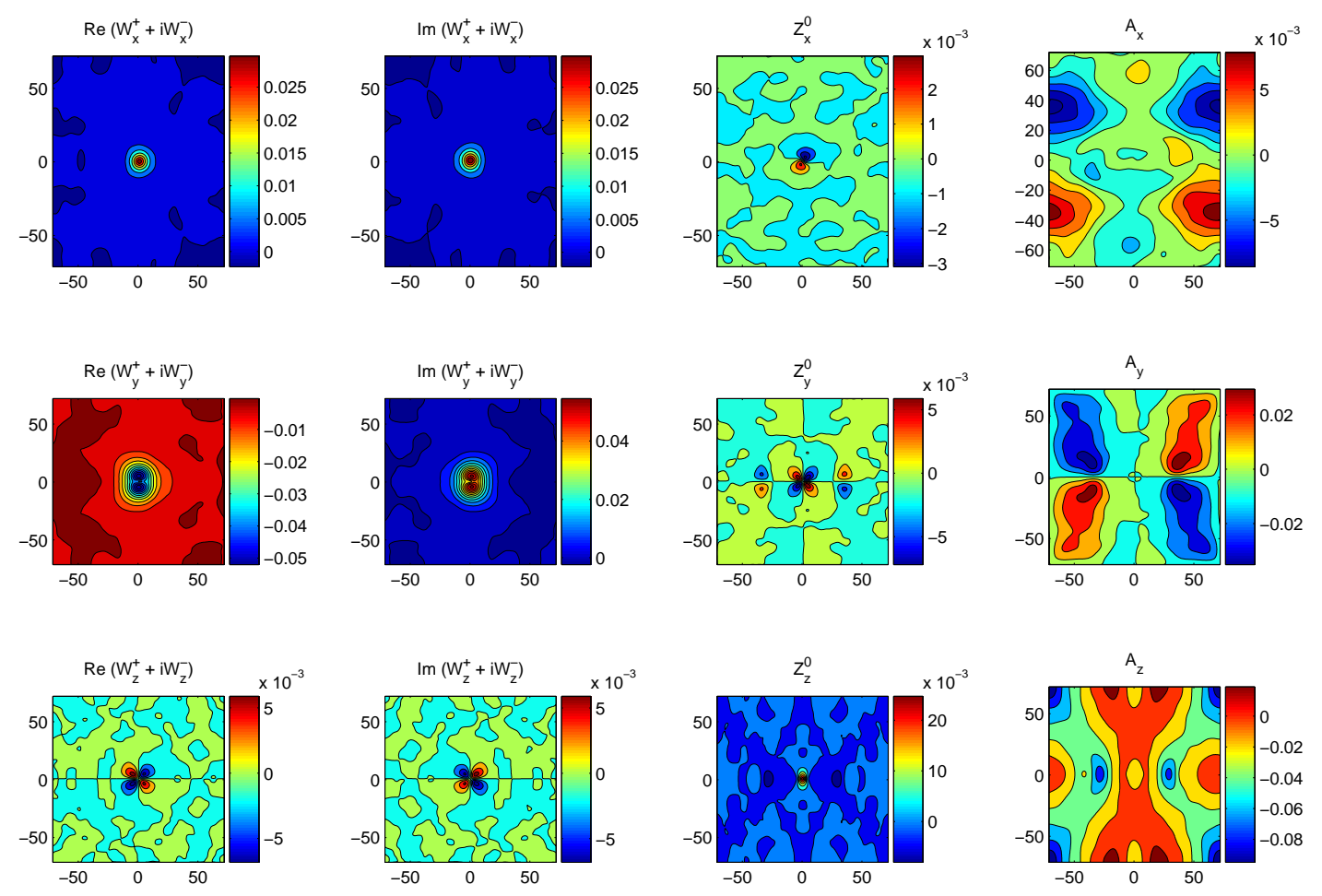

FIG. 5: A snapshot of the gauge fields in the $x=0$ plane for the simulation of Fig. 1 at time $t=50,000$. Subscripts refer to spatial components.

To illustrate the field configurations that make up the oscillon, we graph the fields at time $t=50,000$ for the two-dimensional slice $x=0$. Fig. 5 shows the gauge field components. It is most illustrative to consider a linear superposition of the $W_{j}^{ \pm}$fields, as shown in the figure. Fig. [6 shows the electric fields, which are given by the time derivatives of the gauge fields for our choice of gauge. Fig. 7 shows the components of the Higgs field and its first time derivative, and Fig. 8 shows the magnitude of the Higgs field and the first time derivative of this quantity, together with the total energy density. The oscillon is constructed primarily out of the lower component of the Higgs field, the imaginary part of the upper component of the Higgs field, the $x$ and $y$ spatial components of the $W_{j}^{ \pm}$fields, and the $z$ spatial component of the $Z_{j}^{0}$ field. We see the multipole structures we anticipated from the spherical ansatz analysis. The Higgs field contains monopole and dipole fluctuations. The photon field $A_{j}$ contains delocalized background radiation that was emitted as the oscillon formed from the initial conditions. As we would expect from Eq. (23), it has a dipole structure. In the spherical ansatz, the $W_{j}^{ \pm}$and $Z_{j}^{0}$ fields can potentially contain monopole, dipole, and quadrupole components. Here we see significant monopole and quadrupole structures, but only a very small dipole component, which appears in $Z_{j}^{0}$. As a result, the electric charge we estimate from Eq. (23) is very small, as is the 

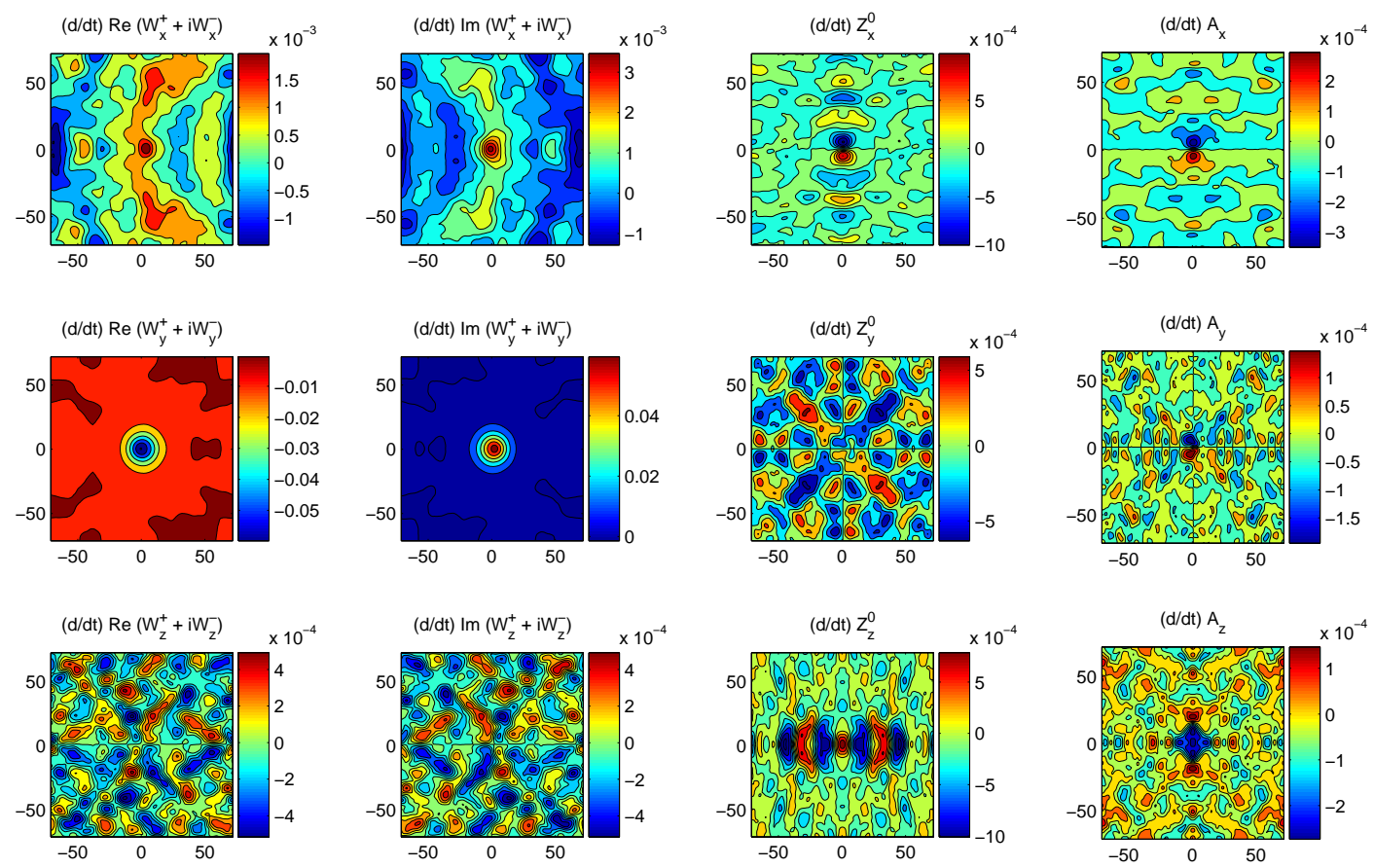

FIG. 6: A snapshot of the electric fields (time derivatives of the gauge potentials) in the $x=0$ plane for the simulation of Fig. 1 at time $t=50,000$. Subscripts refer to spatial components.
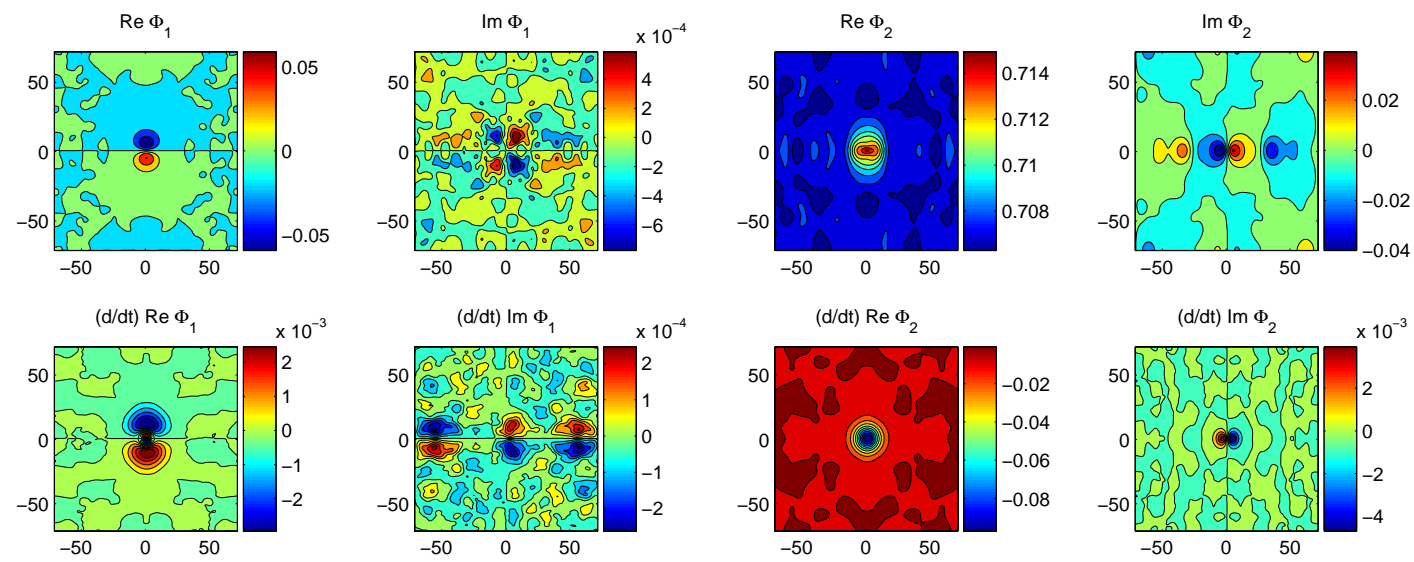

FIG. 7: A snapshot of the Higgs field and its time derivatives in the $x=0$ plane for the simulation of Fig. 1 at time $t=50,000$. Subscripts refer to components of the Higgs field.

true value from the numerical simulation; the oscillon is decoupled from the electromagnetic background. ${ }^{3}$

Each excited field oscillates at a frequency just below its mass. In our units, these oscillations have typical amplitude of order 0.1 and typical radius of order 10. By comparing the total number of cycles to the total time, we find

\footnotetext{
${ }^{3}$ While the multipole analysis is instructive as a description of the field configuration, is is important to note that because the oscillon has large spatial extent compared to its period of oscillation, it is in exactly the domain where the standard multipole expansion for the electromagnetic radiation emitted is invalid.
} 

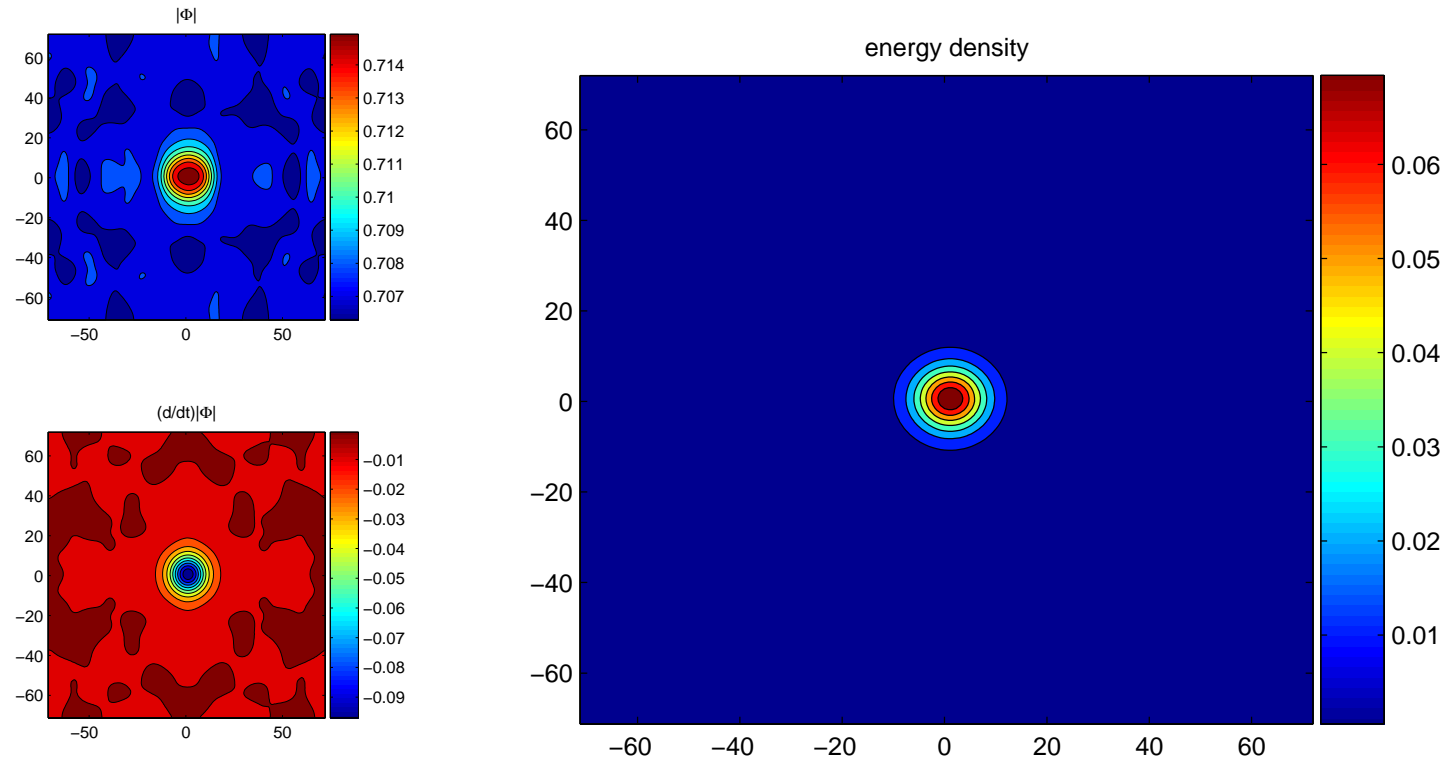

FIG. 8: Left panel: A snapshot of the magnitude of $\phi$ and its first time derivative in the $x=0$ plane for the simulation of Fig. 1 at time $t=50,000$. Right panel: A snapshot of the energy density the $x=0$ plane for the simulation of Fig. 1 at time $t=50,000$.
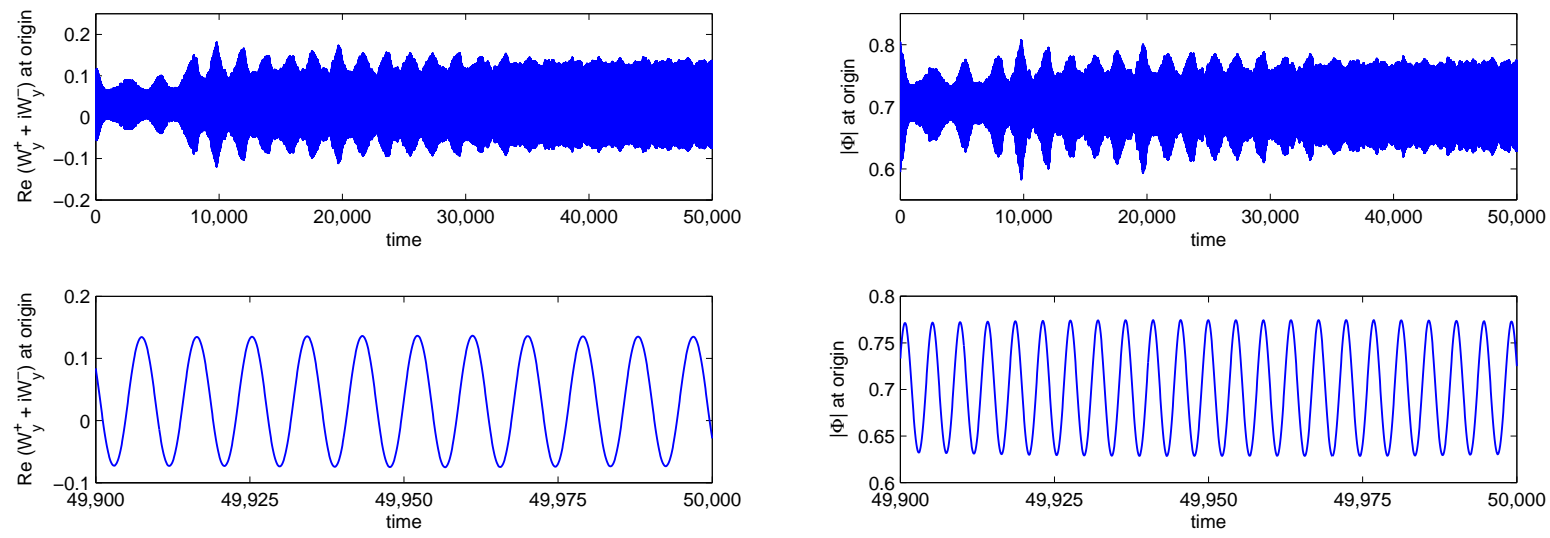

FIG. 9: Oscillon fields at the origin as functions of time. The left side shows one component of the $S U(2)$ gauge field. The upper graph shows the full extent of the simulation. On this scale, the individual oscillations are too small to be seen. Instead, we see the decaying beat pattern from the transient "breathing" motion. The lower graph shows the oscillation of the field for a short time at the end of the simulation (when the transient effects have decayed away). The right side shows the magnitude of $\Phi$ in the same way. It oscillates with fundamental frequency twice that of the gauge field.

$\omega_{H}=1.404$ for the Higgs field components and $\omega_{W}=0.702$ for the gauge field components. These properties are all very similar to the spherical ansatz oscillon. They are also consistent with a small-amplitude analysis, as described in the Introduction, with $\epsilon$ of order 0.1. In Fig. 9, oscillon fields at the origin are shown as functions of time. The fundamental oscillation of each field is modulated by the decaying beat pattern.

The oscillon we have seen is not significantly altered by small perturbations of the initial conditions. As an example, in Fig. 10 we show the results of a run in which the rotational symmetry has been explicitly broken. We take initial conditions as before, except we introduce different rescalings of the $x, y$ and $z$ coordinates in the definition of $\boldsymbol{r}$. As an additional numerical check, this run also uses a smaller time step, $\Delta t=0.05$. Although the beat pattern is slightly enhanced, likely indicating that we have started further away from the true oscillon because of the 


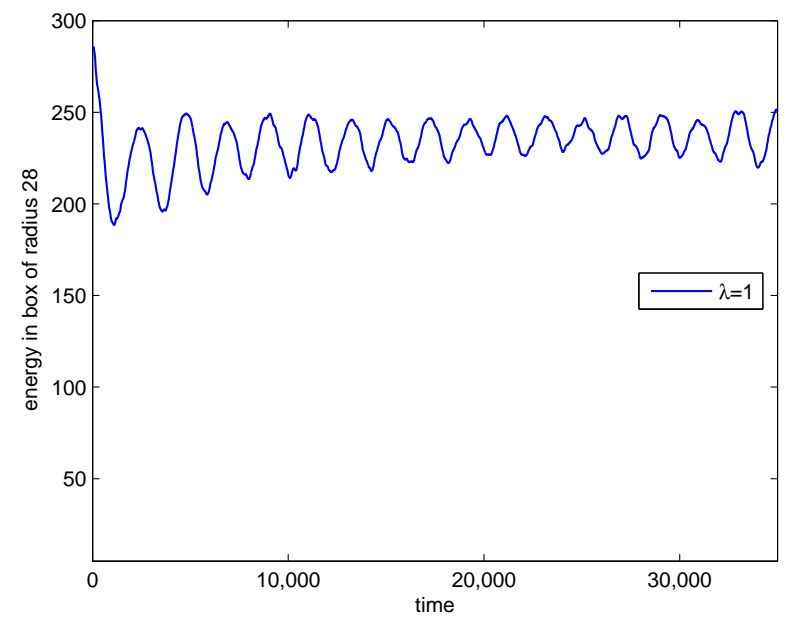

FIG. 10: Energy in a box of radius 28 as in Fig. 1 but with initial conditions that have been deformed to break rotational symmetry. The spatial coordinate $\boldsymbol{r}=x \hat{\boldsymbol{x}}+y \hat{\boldsymbol{y}}+z \hat{\boldsymbol{z}}$ has been everywhere replaced by $\boldsymbol{r}^{\prime}=0.98 x \hat{\boldsymbol{x}}+1.02 y \hat{\boldsymbol{y}}+0.97 z \hat{\boldsymbol{z}}$ and similarly $r$ and $\hat{\boldsymbol{r}}$ have been replaced by $r^{\prime}=\left|\boldsymbol{r}^{\prime}\right|$ and $r^{\prime}=\boldsymbol{x}^{\prime} / \boldsymbol{r}^{\prime}$. As a further check of the numerics, this run also uses a smaller time step, $\Delta t=0.05$. Except for these modifications, the simulation is the same as in Fig. 1.

nonspherical deformation, we see that the system nonetheless converges to a very similar configuration to the case without the rescaling. Equivalent behavior is seen when we make these two changes individually and when we make other perturbations, such as variations of $\chi$.

Finally, we consider the topological properties of the electroweak oscillon. Unfortunately, as shown in [47], there is no unambiguous definition of the topological charge for solutions to the equations of motion. (Topological properties are typically studied using vacuum-to-vacuum paths [48], which are clearly not solutions to the equations of motion since they do not conserve energy.) However, for any localized spatial configuration in which the Higgs field never vanishes, the Higgs winding number is unambiguously defined as

$$
n=\frac{1}{24 \pi^{2}} \int \epsilon_{i j k} \operatorname{Tr}\left[U^{\dagger}\left(\partial_{i} U\right) U^{\dagger}\left(\partial_{j} U\right) U^{\dagger}\left(\partial_{k} U\right)\right] d^{3} x,
$$

where $U$ is the unique $S U(2)$ matrix associated with a nonvanishing Higgs field $\Phi$, so that

$$
\Phi=|\Phi| U\left(\begin{array}{l}
0 \\
1
\end{array}\right) .
$$

The Higgs winding number is a topological invariant, which can only change with time if the Higgs field passes through zero at some point in space. The change in the Higgs winding is physically meaningful and measures whether the fields have crossed the sphaleron barrier. Because the electroweak oscillon contains only small-amplitude field fluctuations, its Higgs winding is always zero and it does not approach the sphaleron barrier. Correspondingly, its topological density

$$
q=\frac{g^{2}}{64 \pi^{2}} \epsilon^{\mu \nu \lambda \sigma} \boldsymbol{F}_{\mu \nu} \cdot \boldsymbol{F}_{\lambda \sigma}
$$

is small as well. But the restriction to small amplitude does not apply to its decays (induced, for example, by collision with another oscillon), when the fields frequently exhibit an implosion to small radii and large amplitudes before ultimately dispersing. This behavior is seen in Fig. 3 for the oscillon's decay when $\lambda$ is slightly less than one. However, both this particular decay and limited experiments with oscillon collisions have not led to winding in the final Higgs field. Current work continues to investigate this possibility.

\section{CONCLUSIONS}

We have seen in detail the results of a numerical simulation describing a long-lived, localized, oscillatory solution to the equations of motion in the bosonic sector of the electroweak Standard Model, for a Higgs mass that is twice the 
$W^{ \pm}$mass. Compared to the natural scales of the system, this solution has small field amplitudes, large spatial extent, and large total energy. In the quantized theory, it would represent a coherent superposition of many elementary particles, and thus is well described by the classical analysis undertaken here. Quantization of the small oscillations around the classical solution would nonetheless be of interest, as has been done for $Q$-ball oscillons in [49]. It would also be desirable to incorporate fermion couplings, which have been ignored here. Such an analysis would require introducing chiral fermions on the lattice, which is well known to be a difficult problem, but one on which significant progress has been made in recent years. While one might expect the oscillon to be destabilized by decay to light fermions, in the case of the photon coupling we have seen that the analogous decay mechanism is highly suppressed.

Because it would require bringing many Higgs and gauge particles together at once, forming such an oscillon would likely require large energies available only in the early universe. If extremely long-lived, such an oscillon could be a dark matter or ultra-high energy cosmic ray candidate. A slow fermion decay mode would be of interest for baryogenesis, since it could provide a mechanism for fermions to be produced out of equilibrium, as is necessary to avoid washout of particle/antiparticle asymmetry. The oscillon has small amplitude everywhere and thus remains far from the sphaleron configuration, even though it has energy above the height of the sphaleron barrier. However, when induced to decay, for example by a collision with another oscillon, the fields typically collapse to a configuration with small radius and large energy density and field amplitudes before dispersing. Such decays could potentially cross the sphaleron barrier and produce fermion number violation. For baryogenesis applications, one would also need to incorporate interactions containing $C$ and $C P$ violation in the classical effective action.

The spherical ansatz provided a crucial tool for obtaining the electroweak oscillon solution. However, any search for oscillons using a particular ansatz cannot guarantee that all solutions have been found. "Emergent" techniques, in which oscillons form from generic initial conditions, offer the opportunity for more comprehensive searches for oscillons, albeit at a higher computational cost. In simpler models, oscillons have been shown to emerge from phase transitions [30] and from thermal initial conditions in an expanding universe [50]. Clearly, it would be desirable to extend these techniques to the electroweak model.

The electroweak oscillon remains stable even when one would expect it to decay, suggesting that there might exist other stable, oscillatory solutions in the electroweak theory or its extensions, either for generic or specific mass ratios. While results for generic mass ratios are clearly of broader applicability, a compelling result for a specific mass ratio might suggest a preferred value of the Higgs mass.

\section{ACKNOWLEDGMENTS}

It is a pleasure to thank E. Farhi, F. Ferrer, M. Gleiser, A. Guth, R. R. Rosales, R. Stowell, J. Thorarinson, and T. Vachaspati for helpful discussions, suggestions and comments; P. Lubans, C. Rycroft, S. Sontum, and P. Weakleim for Beowulf cluster technical assistance; and the Massachusetts Institute of Technology (MIT) Center for Theoretical Physics for hospitality and support while this work was being carried out. N. G. was supported by National Science Foundation (NSF) grant PHY-0555338, by a Cottrell College Science Award from Research Corporation, and by Middlebury College.

Computational work was carried out on the Hewlett-Packard (HP) Opteron cluster at the California NanoSystems Institute (CNSI) High Performance Computing Facility at the University of California, Santa Barbara (UCSB), supported by CNSI Computer Facilities and HP; the Hoodoos cluster at Middlebury College; and the Applied Mathematics Computational Lab cluster at MIT. Access to the CNSI system was made possible through the UCSB Kavli Institute for Theoretical Physics Scholars Program, which is supported by NSF grant PHY99-07949.

* Electronic address: ngraham@middlebury.edu

[1] S. Coleman, Aspects of Symmetry (Cambridge University Press, 1985).

[2] R. Rajaraman, Solitons and Instantons (North-Holland, 1982).

[3] A. Achucarro and T. Vachaspati, hep-ph/9904229, Phys. Rept. 327 (2000) 347.

[4] M. S. Volkov, hep-th/0609112, Phys. Lett. B644 (2007), 203.

[5] L. Stenflo and M. Y. Yu, Phys. Fluids B1 (1989) 1543.

[6] R. F. Dashen, B. Hasslacher and A. Neveu, Phys. Rev. D11 (1975) 3424.

[7] S. Coleman, Nucl. Phys. B262 (1985) 263.

[8] D. K. Campbell, J. F. Schonfeld and C. A. Wingate, Physica 9D (1983) 1.

[9] H. Segur and M. D. Kruskal, Phys. Rev. Lett. 58 (1987) 747. 
[10] N. Graham and N. Stamatopoulos, hep-th/0604134, Phys. Lett. B639 (2006) 541.

[11] M. Gleiser and A. Sornborger, patt-sol/9909002, Phys. Rev. E62 (2000) 1368.

[12] M. Hindmarsh and P. Salmi, hep-th/0606016, Phys. Rev. D74 (2006) 105005.

[13] C. Rebbi and R. Singleton, Jr., hep-ph/9601260, Phys. Rev. D54 (1996) 1020; P. Arnold and L. McLerran, Phys. Rev. D37 (1988) 1020.

[14] M. Gleiser and J. Thorarinson, hep-th/0701294.

[15] I. L. Bogolyubsky and V. G. Makhankov, JETP Lett. 24 (1976) 12.

[16] M. Gleiser, hep-ph/9308279, Phys. Rev. D49 (1994) 2978; E. J. Copeland, M. Gleiser and H. R. Muller, hep-ph/9503217, Phys. Rev. D52 (1995) 1920; A. Adib, M. Gleiser and C. Almeida, hep-th/0203072, Phys. Rev. D66 (2002) 085011.

[17] E. P. Honda and M. W. Choptuik, hep-ph/0110065, Phys. Rev. D65 (2002) 084037.

[18] S. Kasuya, M. Kawasaki and F. Takahashi, hep-ph/0209358, Phys. Lett. B559 (2003) 99.

[19] G. Fodor, P. Forgács, P. Grandclément and I. Rácz, hep-th/0609023, Phys. Rev. D74 (2006) 124003.

[20] B. Piette and W. J. Zakrzewski, Nonlin. 11 (1998) 1103.

[21] M. Gleiser, hep-th/0408221, Phys. Lett. B600 (2004) 126; P. M. Saffin and A. Tranberg, hep-th/0610191

[22] A. Kusenko, hep-th/9704073, Phys. Lett. B404 (1997) 285.

[23] A. Kusenko and M. E. Shaposhnikov, hep-ph/9709492, Phys. Lett. B418 (1998) 46.

[24] K. Enqvist and J. McDonald, hep-ph/9711514, Phys. Lett. B425 (1998) 309.

[25] S. Kasuya and M. Kawasaki, hep-ph/9909509, Phys. Rev. D61 (2000) 041301.

[26] E. W. Kolb and I. Tkachev, astro-ph/9311037, Phys. Rev. D49 (1994) 5040.

[27] M. Broadhead and J. McDonald, hep-ph/0503081, Phys. Rev. D72 (2005) 043519.

[28] A. Rajantie and E. J. Copeland, hep-ph/0003025, Phys. Rev. Lett. 85 (2000) 916;

[29] M. Gleiser, hep-th/0602187.

[30] M. Gleiser and R. C. Howell, hep-ph/0209176, Phys. Rev. E68 (2003) 065203(R); hep-ph/0409179, Phys. Rev. Lett. 94 (2005) 151601.

[31] G. Fodor and I. Rácz, hep-th/0311061, Phys. Rev. Lett. 92 (2004) 151801; P. Forgács and M. S. Volkov, hep-th/0311062, Phys. Rev. Lett. 92 (2004) 151802; G. Fodor and I. Rácz, hep-th/0609110

[32] J. N. Hormuzdiar and S. D. Hsu, hep-ph/9805382, Phys. Rev. C59 (1999) 889.

[33] I. Dymnikova, M. Yu. Khlopov, L. Koziel and S. G. Rubin, hep-th/0010120, Grav. Cosm. 6 (2000) 311.

[34] N. Graham, hep-th/0610267, Phys. Rev. Lett. 98 (2007) 101801.

[35] N. F. Lepora, hep-th/0210018, Phys. Lett. B541 (2002) 362.

[36] E. Farhi, N. Graham, V. Khemani, R. Markov and R. R. Rosales, hep-th/0505273, Phys. Rev. D72 (2005) 101701.

[37] R. F. Dashen, B. Hasslacher and A. Neveu, Phys. Rev. D10 (1974) 4138; E. Witten, Phys. Rev. Lett. 38 (1977) 121; P. Forgács and N. S. Manton, Commun. Math. Phys. 72 (1980) 15; B. Ratra and L. G. Yaffe, Phys. Lett. B205 (1988) 57.

[38] R. Stowell, E. Farhi, N. Graham, A. Guth, and R. R. Rosales, work in preparation.

[39] A. M. Kosevich and A. S. Kovalev, Zh. Eksp. Teor. Fiz. 67 (1975) 1793; R. R. Rosales, unpublished research notes.

[40] K. Huang, Quarks, Leptons and Gauge Fields (World Scientific, 1992).

[41] K. Wilson, Phys. Rev. D10 (1974) 2445.

[42] J. Smit, Introduction to Quantum Fields on a Lattice, (Cambridge University Press, 2001).

[43] J. Ambjorn, T. Askgaard, H. Porter and M. E. Shaposhnikov, Nucl. Phys. B353 (1991) 346.

[44] A. Tranberg and J. Smit, hep-ph/0310342, JHEP 0311 (2003) 016.

[45] A. Rajantie, P. M. Saffin and E. J. Copeland, hep-ph/0012097, Phys. Rev. D 63 (2001) 123512.

[46] R. Stowell, unpublished research notes.

[47] E. Farhi, J. Goldstone, S. Gutmann, K. Rajagopal and R. L. Singleton, hep-ph/9410365, Phys. Rev. D51 (1995) 4561.

[48] F. R. Klinkhamer and N. S. Manton, Phys. Rev. D30 (1984) 2212.

[49] N. Graham, hep-th/0105009, Phys. Lett. B513 (2001) 112.

[50] E. Farhi, N. Graham, A. Guth, N. Iqbal, R. Rosales and N. Stamatopoulos, work in preparation. 